\title{
Electrocortical and Hemodynamic Changes within the Brain during Incremental Bicycle Exercise in Normoxia and Hypoxia-A Combined EEG/NIRS Study
}

\author{
Vera Abeln ${ }^{1}$, Stefan Schneider ${ }^{1,2}$, Axel Knicker ${ }^{1}$, Thorsten Schiffer ${ }^{3}$, Wildor Hollmann ${ }^{4}$ and Heiko Klaus Strüder ${ }^{1}$ \\ 1. Institute of Movement and Neurosciences, German Sport University, Cologne 50933, Germany \\ 2. Faculty of Science, Health, Education and Engineering, University of the Sunshine Coast, Queensland 4558, Australia \\ 3. Outpatient Clinic for Sports Traumatology and Public Health Consultation, German Sport University, Cologne 50933, Germany \\ 4. Institute for Cardiology and Sports Medicine, German Sport University, Cologne, Am Sportpark Müngersdorf 6, 50933 Cologne, \\ Germany
}

\begin{abstract}
The correlation of NIRS (near-infrared spectroscopy) and EEG (electro-cortical activity) in exercise studies has never been shown. Eight sport students performed an incremental bicycle exercise test under normoxic and hypoxic $\left(12.7 \% \mathrm{O}_{2}\right)$ conditions respectively. EEG and NIRS recordings of the prefrontal cortex (PFC, Brodmann area 10. 46) were performed synchronously to shed light on their correlation. ANOVA revealed a higher absolute workload $(231.3 \pm 37.2 \mathrm{~W})$, and relative PFC oxygenation under normoxic conditions, whereas hypoxic conditions resulted in earlier exhaustion $(200 \pm 26.7 \mathrm{~W})$ and reduced PFC oxygenation. NIRS parameters increased remarkably with exercise intensity $(P<0.001)$ and differed between conditions $\left(\mathrm{O}_{2} \mathrm{Hb}: P<0.001 ; \mathrm{HHb}: P=\right.$ 0.023 ; tHb: $P=0.016)$ and hemispheres $\left(\mathrm{O}_{2} \mathrm{Hb}: P=0.023\right)$. For EEG, higher prefrontal cortical current density during compared to pre and post exercise was revealed for both conditions $(P<0.001)$. No difference between conditions and hemispheres were found. In conclusion, brain cortical activity is not impaired by hypoxia. No correlation between NIRS and EEG, but a moderate correlation between EEG and cardio-vascular parameters and a moderate to high correlation between NIRS and cardio-vascular parameters were found. The results emphasize that the transfer of EEG and NIRS results need to be done with caution.
\end{abstract}

Key words: Cerebral oxygenation, central fatigue, cortical current density, prefrontal cortex.

\section{Introduction}

The human brain function is widely oxygen dependent. If and how brain activity is affected by oxygen limitations during exercise is unclear, because it is still challenging to display acute cerebral changes during exercise due to the susceptibility of brain imaging methods to movement artefacts. Developments of more flexible and robust techniques like EEG (electroencephalography) using active electrodes or NIRS (near-infrared spectroscopy) now promise to permit examining changes in

Corresponding author: Vera Abeln, Ph.D., research associate, research fields: exercise science, neuro-physiology, brain imaging and psychophysiology. E-mail: v.abeln@dshs-koeln.de. electro-cortical and hemodynamic situation within the brain.

While EEG records electro-cortical activity directly from the scalp, NIRS is used to measure it indirectly via concentration changes of $\mathrm{O}_{2} \mathrm{Hb}$ (oxygenated), $\mathrm{HHb}$ (deoxygenated) and $\mathrm{tHb}$ (total haemoglobin) in brain tissue [1]. Although, NIRS theoretically allows assessing of all cortical brain regions, in exercise studies, due to handling reasons, the PFC (prefrontal cortex) has predominantly been regarded. Using NIRS, investigations of cerebral hemodynamic changes during incremental exercise have shown increasing prefrontal oxygenation with exercise intensity [2, 3] and decreasing $\mathrm{O}_{2} \mathrm{Hb}$, increasing $\mathrm{HHb}$ and stable $\mathrm{tHb}$ concentrations at the end of exhaustive exercise within 
the PFC [4-6].

In hypoxia, when oxygen availability is limited, cerebral oxygenation was found to decrease with hypoxia level under resting conditions already [7]. During exercise in hypoxia, lower $\mathrm{O}_{2} \mathrm{Hb}$ and higher $\mathrm{HHb}$ were observed with increasing exercise intensity, while $\mathrm{tHb}$ was reduced [2, 3, 8-11].

Based on the fact that the PFC is associated with cognitive tasks, motivation and decision-making, decreasing oxygenation at high exercise intensity prior to exhaustion in the PFC has been assumed to trigger centrally mediated fatigue and the decision to stop exercise in numerous former studies [3, 6, 9, 12-15]. Hypoxia is proposed to accelerate cerebral deoxygenation, thus central fatigue $[2,8,10,11,15$, 16]. The underlying mechanism of central fatigue is widely unknown at present. However, as NIRS is supposed to assess changes in neural activity, PFC deoxygenation is presumed to reflect impaired PFC activity and by this may disturb motor output $[3,4,6$, $13,17,18]$.

Using EEG, even less research has been done regarding electro-cortical changes in hypoxia or during exercise. Many former studies during exercise are difficult to interpret due to: use of single electrodes; the missing localization, but multi-functional nature of the brain; heterogeneous findings of changes in frequency bands [19]. Applying a complete 32-electrode system and analysing total cortical current density, increased cortical current density within the primary motor cortex with exercise intensity was found; whereas, no significant enhancements of activity within the PFC have been identified [20]. The found stable EEG activity within the PFC during exercise is contradictory to the found changes of NIRS studies reported earlier. Therefore, a correlation of EEG and NIRS has to be questioned. Moreover, the fundamental linear relationship of hemodynamic and metabolic changes of the neurovascular coupling principle, on which NIRS is based on, has meanwhile been shown to be non-linear and locally different $[21,22]$. No study investigated electro-cortical changes during exercise in hypoxia so far. Thus, it is unclear whether PFC activity is impaired when oxygen availability is reduced and if this contributes to earlier occurring fatigue. Furthermore, hemispheric differences in the PFC have been shown earlier in regard to exercise and mood [12]. Irrespective of these hemispheric differences, most previous NIRS studies in regard to exercise neglected to differentiate changes between hemispheres.

Accordingly, this study aimed to measure both, electro-cortical current density and hemodynamic changes synchronously, and within both PFC hemispheres, during an incremental bicycling exercise test using a combination of NIRS and EEG. It is hypothesized that NIRS, but not EEG parameters, increase within the PFC in the course of an incremental exercise test; and hemispheric differences will be found. Moreover, it is hypothesized that in hypoxia, performance will be impaired, and PFC oxygenation is lower than in normoxia accompanied by impaired PFC activity. Hemispheric changes will be measured with EEG and NIRS to shed light on the correlation between electrocortical and hemodynamic changes.

\section{Experimental Procedures}

\subsection{Participants}

Ten healthy male sport students (age $27 \pm 4.2$ years; weight $79 \pm 12.6 \mathrm{~kg}$; height $180 \pm 3.9 \mathrm{~cm}$ ) voluntarily took part in this study. Volunteers have been informed about the subject and potential risks of the study. All accepted and signed a written informed consent approved by the local ethic committee in accordance with the Declaration of Helsinki. None of the volunteers suffered from any neurological or medical problems or diseases, which would have consequences on exercise performance or neurophysiological measurements.

Participants have randomly been assigned to two test groups. The first group performed an incremental bicycle exercise test, first under normoxic conditions $\left(21.0 \% \mathrm{O}_{2}\right)$, and at the second test day under hypoxic 
conditions $\left(12.7 \% \quad \mathrm{O}_{2}\right.$, simulating acute altitude exposure of $4000 \mathrm{~m}$ ). The second group completed the two exercise tests in reversed order. Test days were separated on average by $25 \pm 16.6$ days. All tests were carried out in a temperature-controlled laboratory. Beside from wearing silicon breathing half mask (x-plore 4740, DRÄGER AG \& Co. KGaA, Lübeck, Germany) over nose and mouth for administration of the hypoxic gas mixture (starting 15 minutes prior to the exercise protocol until the end of the test), participants completed the same protocol on the two test days. Oxygen saturation was shown reduced by about $10 \%-15 \%$ after 10 minutes of $12.7 \% \mathrm{O}_{2}$-hypoxic gas exposure [23].

\subsection{Exercise Protocol}

A continuous incremental exercise test on a bicycle ergometer (Ergoliner 900, ERGOLINE, Bitz, Germany) commenced at 50 Watt $(\mathrm{W})$, increased every 5 minutes by $50 \mathrm{~W}$ until subjective exhaustion or up to a heart rate of maximally 180 beats per minute was conducted. The heart rate limit was set at 180 beat per minute in order to keep the strain below the cardio circulatory and metabolic maximum to avoid potential complications of acute oxygen deficit, such as orthostatic collapse, cardiac arrhythmia, fainting, etc. Pedalling cadence was to be kept constant during the tests at $70 \pm 5$ rotations per minute provided to the athletes by a visible digital display at the handlebar of the ergometer.

\subsection{Heart Rate, Lactate and Ratings of Perceived Exertion}

Before the start and during the last minute of each exercise stage, the heart rate was measured (Polar S810i, POLAR ELECTRO, Buettelborn, GER). Ratings of perceived exertion [RPE, 24] were requested at the end of each stage. 20- $\mu$ l capillary bloods from the earlobe for lactate analysis (BiosenC_Line Glucose-/Lactate Measurement System-EKF DIAGNOSTICS, Barleben, GER) were taken prior to exercise and during the last minute of each exercise stage.

\subsection{EEG (Electroencephalography)}

Information about set-up and processing of EEG is published in Abeln, Harig, Knicker, Vogt \& Schneider [25] unless otherwise noted. Prior to and approximately five minutes after a passive break following the exercise protocol a 1-minute rest-EEG was performed. Therefore, participants were asked to sit relaxed on the bicycle ergometer, arms resting on the handlebar and feet on the pedals, to keep their eyes closed. Raw data was filtered using Butterworth Zero Phase Filters including a notch filter at $50 \mathrm{~Hz}$, low cut-off at $2 \mathrm{~Hz}$ and high cut-off at $70 \mathrm{~Hz}$ with a time constant of $0.1592 \mathrm{sec}$ and 48 decibel per octave. Based on triggers, data was afterwards segmented into the rest measurement prior to (pre) and after the exercise test (post), as well as each exercise stage $(50 \mathrm{~W}, 100 \mathrm{~W}$, etc.), of which at least 1 minute has been completed) without overlap. Minute 5 of each exercise stage was excluded from analysis because of distraction and interruption within brain activity due to taken heart rate, lactate and ratings of perceived exertion assessments. Loreta analysis was used in order to export the cortical current density $\left[\mu \mathrm{V}^{2} / \mathrm{mm}^{4}\right]$ within the anterior prefrontal cortex (Brodmann area 10 (BA10, superior + middle + medial + inferior frontal gyrus) and within the dorsolateral prefrontal cortex (BA46, inferior + middle frontal gyrus). The latter two areas correspond to the investigated region by NIRS and were pooled as PFC, which was regarded in most of the former studies due to handling reasons (no hair, easy to fix probes etc.). We refrained from functional differentiation of the two regions of interest, because the behaviour/role of both BA are quite similar and/because the number of EEG channels is low.

\subsection{NIRS (Near-Infrared Spectroscopy)}

$\mathrm{O}_{2} \mathrm{Hb}, \mathrm{HHb}$ as well as $\mathrm{tHb}\left(\mathrm{O}_{2} \mathrm{Hb}+\mathrm{HHb}\right)$ were recorded over the left and right $\mathrm{PFC}$ of the brain with a 
frequency of $50 \mathrm{~Hz}$ using a NIRS system (Oxymon, Artinis, NL). Black near-infrared light emitter(wavelength: 764 and $856 \mathrm{~nm}$ ) and receiver(emitter-receiver distance: $4.5 \mathrm{~cm}$ ) probe holders were integrated into the EEG cap at positions AF7 and AF3 for left and at AF8 and AF4 for right hemisphere of a 64-channel EEG-cap above BA10 and BA46. Forehead skin has been cleaned with alcohol-impregnated pads before. Special care was taken for the correct and secure fit of the sensors and double-sided adhesive tape underneath the probe holders was used to avoid sensor shift. For age dependent differences in the scattering medium of human tissue, differential path length factor was individually calculated based on participants age [26].

At first data has been baseline corrected. Based on triggers, data were then segmented into rest (pre, post) and exercise measurements $(50 \mathrm{~W}, 100 \mathrm{~W}, 150 \mathrm{~W}, 200$ $\mathrm{W}$ etc.). The mean of the $\mathrm{O}_{2} \mathrm{Hb}, \mathrm{HHb}$ and $\mathrm{tHb}[\mu \mathrm{mol} / \mathrm{l}]$ of a 60 -second period starting from the trigger pre and post were exported. For during exercise, minutes 1 to 4 of each stage starting from the exercise stage trigger were exported. Minute 5 of each stage was excluded for the same reason as for EEG analysis. Segments that have visibly been affected by movement artefacts were rejected.

\subsection{Statistics}

Eight participants were included into the analysis ( $N$ $=8$ ). Two volunteers had to be rejected from the analysis, because one quit the test under hypoxic conditions after two stages due to dizziness, and the data set of the second was incomplete. In order to compare similar level of strain and fatigue (same Watt load was perceived harder and test ended earlier under hypoxic conditions), the last three stages of each trial were averaged and exported. These averaged three final exercise stages, plus the pre- and postmeasurement stages, were included into the statistical analysis.

Statistics were run using the Statistica 7.1 software
(STATSOFT INC., Tulsa, USA). Two-way repeated measures ANOVA (analysis of variance) was used to assess changes in heart rate and lactate concentration. The factor TIME was defined by the measurement time points: pre, 3rd last, 2nd last and last exercise stage of the incremental exercise test, as well as post. Factor CONDITION was implemented for normoxic and hypoxic conditions. To compare the means of significant ANOVA effects, Fisher's LSD (Least Significant Difference) post-hoc test was used. The tests were used for EEG and NIRS plus the factor HEMISPHERE (left, right). Non-parametric ratings of perceived exertion values were analysed using a Friedman's ANOVA and, in case of significance, Wilcoxon tests for paired samples. Multiple regression analysis was used to check for correlations between NIRS $\left(\mathrm{O}_{2} \mathrm{Hb}, \mathrm{HHb}, \mathrm{tHb}\right)$, EEG and cardio-vascular parameters (heart rate, lactate concentration) and perceived exertion during exercise. Values of both conditions and hemispheres have been included into the regression analysis. The threshold for significance was set at $P<0.05(*), P<0.01(* *)$ and $P<0.001$ (***).

\section{Results}

In the following "interaction" means three-way interaction between TIME, CONDITION and HEMISPHERE unless otherwise noted.

For five out of eight trials of the normoxic and four out of eight of the hypoxic tests, the exercise protocol were ended as their heart rate exceeded 180 beats/minute, which was the maximal heart rate defined for safety reasons. On average, participants exercising under normoxic conditions reached $231.3 \pm$ $37.2 \mathrm{~W}$ and $200 \pm 26.7 \mathrm{~W}$ under hypoxic conditions respectively. Please again, take into account that the following results represent relative states of intensity during the 3rd last, 2nd last and last exercise stage respectively, and not absolute intensity levels (intensity in hypoxia is on average lower than in normoxia). 


\subsection{Heart Rate, Lactate, Perceived Exhaustion}

For heart rate, ANOVA for repeated measures $(\mathrm{F}(3$, $21)=3.7472)$ revealed a significant effect for TIME $(P$ $<0.001)$ and an interaction $(P=0.027)$. No effect for CONDITION $(P=0.117)$, but slightly higher heart rate values at rest and during exercise in hypoxia up to the 2nd last exercise stage was found (see Fig. 1). Heart rate increased for normoxic and hypoxic conditions from pre to the 3rd last, 2nd last and last exercise stage (for all $P<0.001$ ).

ANOVA including capillary lactate concentration values for the factor TIME and CONDITION $(\mathrm{F}(3,21)$ $=1.651)$ result in a significant effect for TIME $(P<$ $0.001)$, but not CONDITION $(P=0.373)$ and no interaction $(P=0.208)$. Lactate concentration significantly increased for normoxic and hypoxic conditions from the 3 rd last to the 2 nd last $(P=0.005)$ and from the 2nd last to the last stage $(P<0.001$; see Fig. 1).

Friedman's ANOVA (degree of freedom $=5$ ) revealed $\mathrm{CHI}^{2}=37.408$ and $P<0.001$. Wilcoxon Test for nonparametric, paired variables revealed significant higher ratings of perceived exertion values from the $3 \mathrm{rd}$ last to the 2nd last (normoxic $P=0.012$; hypoxic $P=$ 0.012 ) and from the 2 nd last to the last exercise stage (normoxic $P=0.012$; hypoxic $P=0.018$ ). No difference between CONDITIONs was found (3rd last $P=0.402 ;$ 2nd last $P=0.116$; last $P=0.686$ ).

\subsection{NIRS}

$\mathrm{O}_{2} \mathrm{Hb}$ : Concerning $\mathrm{O}_{2} \mathrm{Hb}$ concentration within the PFC, ANOVA for repeated measures revealed a main effect for TIME $(P<0.001)$, HEMISPEHRE $(P=$ $0.023)$ and CONDITION $(P<0.001)$ as well as an interaction $(P=0.002)$ (see Fig. 2).

Regarding the left prefrontal hemisphere, for normoxic conditions $\mathrm{O}_{2} \mathrm{Hb}$ increased from pre-exercise to all other measurement points (for 3rd last $P=0.011$, for other $P<0.001$ ), and from the 2nd last to the last stage $(P=0.008)$. For hypoxic conditions, $\mathrm{O}_{2} \mathrm{Hb}$ only changed from "pre" to "last" $(P=0.033)$ and "post" $(P$

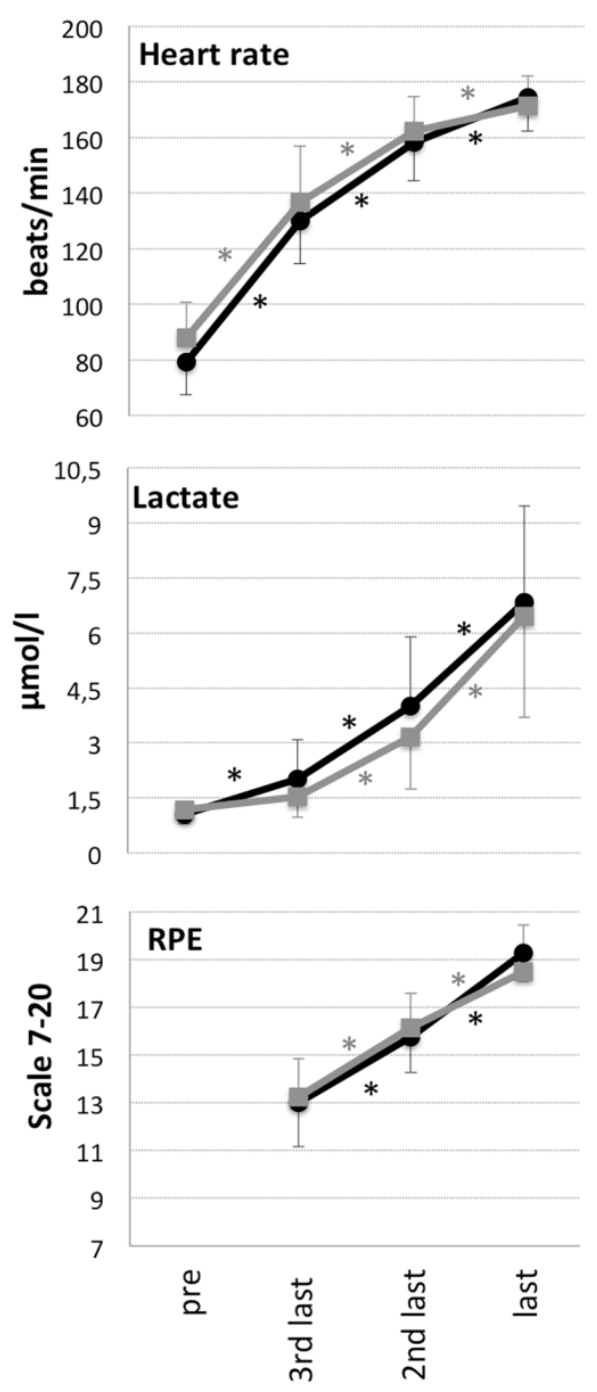

Fig. 1 Results heart rate, lactate concentration and RPE (ratings of perceived exertion). Results are provided for normoxic (black) and hypoxic conditions (grey) for measurement points pre, 3rd last, 2nd last and last exercise stage. Points and squares represent the mean values, bars standard deviations. Significant differences are indicated by $*(P<0.05)$.

$<0.001)$, from the 2 nd last to the last exercise stage ( $P$ $=0.018)$ and from the "last" to "post" exercise $(P=$ 0.011). Beside "pre", all measurements of the left hemisphere under normoxic condition were higher compared to hypoxic condition (for all $(P<0.001)$. Within the right hemisphere, normoxic measurements increased from "pre" to all other measurements $(P<$ $0.001)$, from "3rd last" to "2nd last" $(P=0.005)$ and from "2nd last" to "last" $P<0.001)$. Under hypoxic conditions, "pre" was lower than "post" $(P<0.001)$ 
and "3rd last" lower than "last" $(P<0.011)$. Measurements "3rd last", "2nd last", "last" and "post" of the normoxic condition were higher than the hypoxic measurements (for all $P<0.001$ ). In addition, measurements of the normoxic condition were lower in the left compared to the right hemisphere $(P=0.023)$.

$\mathrm{HHb}$ : In regard to $\mathrm{HHb}$ concentration, a main effect for TIME $(P<0.001)$, CONDITION $(P=0.023)$, but not for HEMISPHERE $(P=0.254)$ and no interaction $(P=0.109)$ was observed (see Fig. 2).

Within the left hemisphere under normoxic conditions, significant increases from "pre" to all measurement points (for 3rd last $P=0.029$, for 2nd last $P=0.003$; for last and post $P<0.001)$ and from " 2 nd last" to "last" $(P=0.032)$ were found. Under hypoxic conditions, increases were observed from "pre" to all measurement points during exercise $(P<0.001)$ and from "2nd last" to "last" $(P=0.005)$. A decrease from "last" to "post" $(P<0.001)$ was revealed. Within the right hemisphere under normoxic conditions, "pre" was lower than all other measurement points (for 3rd last $P=0.001$, for other $P<0.001$ ) and "2nd last" lower than "last" $(P=0.032)$. Under hypoxic conditions, "pre" was lower than all measurements during exercise $(P<0.001)$, "2nd last" lower than "last" $(P=0.045)$ and "last" higher than "post" $(P<0.001)$. Comparing conditions, measurements "3rd last", "2nd last" and "last" were higher under hypoxic conditions than under normoxic conditions (for all $P<0.001$ ) within both hemispheres, whereas for "post" significantly higher values under normoxic than hypoxic conditions have been observed only in the right hemisphere $(P=0.002)$.

tHb: For tHb concentration, a main effect for TIME $(P<0.001)$, CONDITION $(P=0.016)$ as well as an interaction $(P=0.029)$ resulted out of the ANOVA. The factor HEMISPHERE missed level of significance $(P=0.055)$ (see Fig. 2).

In the left prefrontal hemisphere, under normoxic conditions measurement "3rd last" was higher than "pre" $(P=0.028)$, "2nd last" higher than "3rd last" $(P=$
$0.023)$ and "last" higher than "2nd last" $(P=0.001)$. All measurements were higher than "pre" $(P<0.05)$. Higher activity within the left hemisphere under normoxic compared to hypoxic conditions was observed for "2nd last" $(P=0.012)$, "last" $(P=0.006)$ and "post" $(P=0.027)$. Under hypoxic conditions, $\mathrm{tHb}$ increased towards the end of the exercise test from

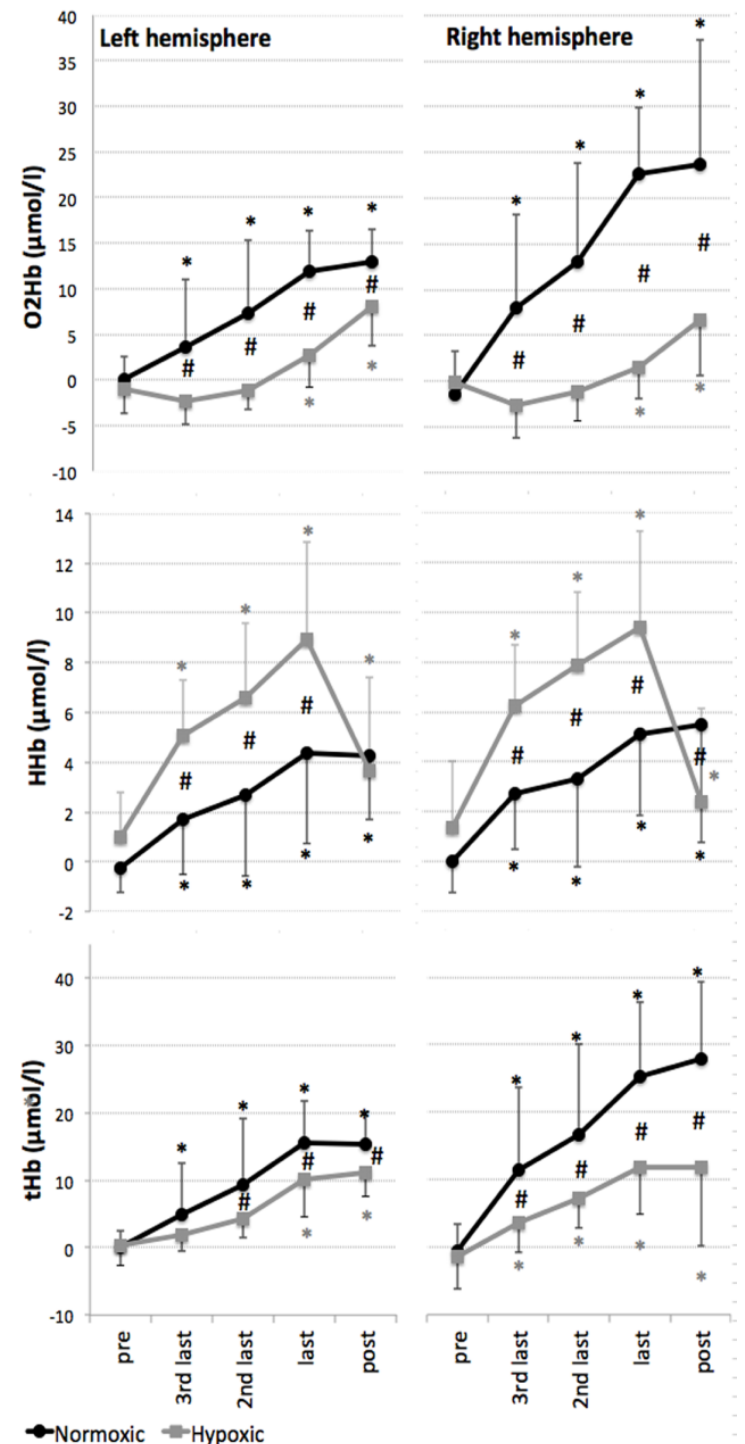

Fig. 2 Results NIRS. Progression of $\mathrm{O}_{2} \mathrm{Hb}$ (top), $\mathrm{HHb}$ (middle) and tHb (bottom) within the left and right hemisphere of the prefrontal cortex for the last three exercise stages (3rd last, 2nd last and last) as well as pre and post exercise under normoxic (black) and hypoxic (grey) conditions. Points and squares represent the mean values, bars standard deviations. Significant differences $(P<0.05)$ between measurement points are indicated by *. Significant differences between conditions are demonstrated by \#. 
"pre" to "last" and "post" $(P<0.001)$ and from "2nd last" to "last" $(P=0.003)$. Within the right hemisphere, tHb increased from "pre" to all other measurement points $(P<0.001)$, from " 3 rd last" to " 2 nd last" $(P=$ $0.010)$ and from "2nd last" to "last" $(P<0.001)$ under normoxic conditions. Under hypoxic conditions, tHb for "pre" was lower compared to all other measurements (for 3rd last $P<0.012$, for other $P<$ $0.001)$. Normoxia revealed significantly higher values compared to hypoxia for measurements "3rd last", "2nd last", "last" and "post" (for all $P<0.001$ ).

\section{$3.3 E E G$}

ANOVA for repeated measures for the activity in the PFC (BA10 + BA46) resulted in a significant main effect for TIME $(P<0.001)$, but neither for CONDITION $(P=0.208)$, HEMISPHERE $(P=0.496)$ nor a three-way interaction ( $P=0.578$, see Fig. 3$)$. An interaction between TIME and HEMISPHERE $(P=$ 0.046) was found.

Regarding normoxia, a main effect of TIME $(P<$ $0.001)$, but not for HEMISPHERE $(P=0.933)$ and no interaction between TIME and HEMISPHERE $(P=$ $0.229)$ was found. Cortical current density during "pre"

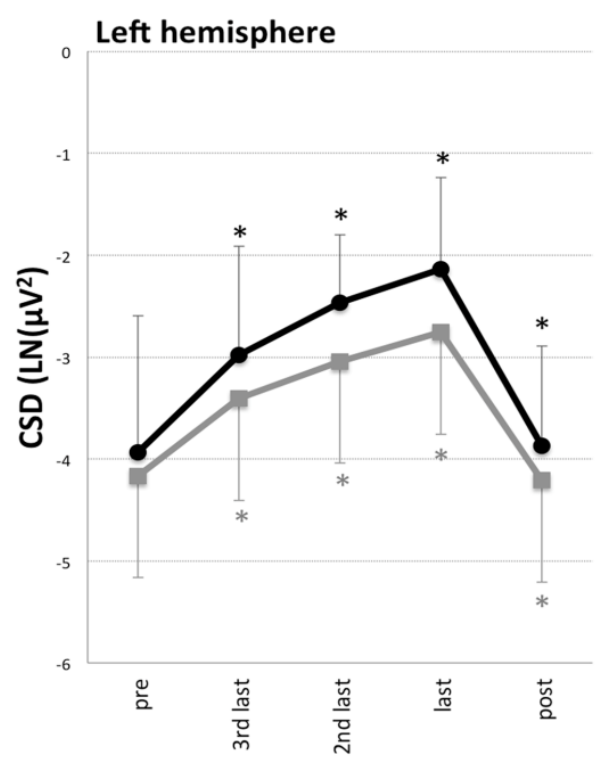

and "post" was significantly lower compared to all measurements during exercise (for 3rd last $P<0.01$; for 2nd last and last $P<0.001)$. In addition, "3rd last" was lower than "last" $(P=0.022)$. For hypoxia, a main effect for TIME $(P<0.001)$ and an interaction between TIME and HEMISPHERE was observed $(P=0.042)$. Significant higher values for "3rd last", "2nd last" and "last" compared to "pre" (to "3rd last" $P=0.01$; to "2nd last" $P<0.001$; to "last" $P<0.001)$ and to "post" (to "3rd last" $P=0.017$; to "2nd last" $P<0.001$; to "last" $P<0.001)$ were found.

\subsection{Correlation}

No correlation was found between NIRS and EEG parameters during exercise (correlation coefficient (R-) and $P$-values are provided in Table 1). EEG did moderately correlate with heart rate and lactate concentration, but not ratings of perceived exertion. $\mathrm{O}_{2} \mathrm{Hb}, \mathrm{HHb}$ and $\mathrm{tHb}$ showed a moderate to high positive correlation with heart rate, lactate and ratings of perceived exertion. Correlations were also found between heart rate, lactate and ratings of perceived exertion as well as between $\mathrm{O}_{2} \mathrm{Hb}$ and $\mathrm{tHb}$ and between $\mathrm{HHb}$ and $\mathrm{tHb}$.

Right hemisphere

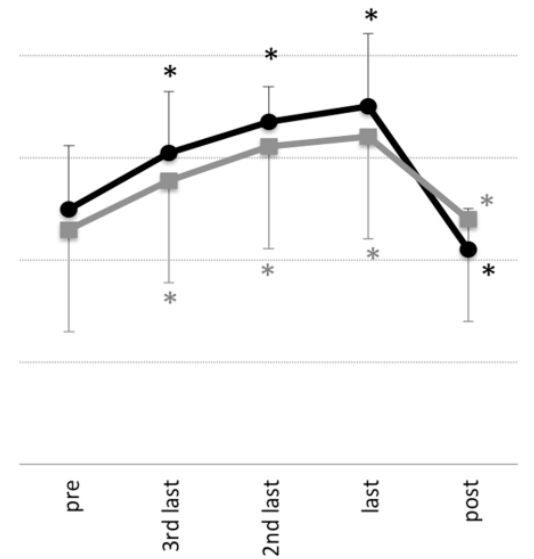

Fig. 3 Results EEG. Progression of CCD (cortical current density) within the left and right hemisphere of the PFC (BA10 + 46) of the 3rd last, 2nd last and last exercise stage as well as pre and post exercise under normoxic (black) and hypoxic (grey) conditions. Points and squares represent the mean values, bars standard deviations. Significant differences $(P<0.05)$ between measurement points are indicated by *. 
Table 1 Correlation. Results of multiple regression analysis for correlation between the NIRS parameters $\mathrm{O}_{2} \mathrm{Hb}($ oxygenated haemoglobin) concentration, HHb (deoxygenated haemoglobin) concentration and tHb (total haemoglobin) concentration, EEG (cortical current density) within the prefrontal cortex as well as HR (heart rate), LAC (lactate concentration) and RPE (ratings of perceived exertion) during exercise. Given are the allocated multiple regression coefficient $(\boldsymbol{R}$-) and $\boldsymbol{P}$-values.

\begin{tabular}{|c|c|c|c|c|c|c|}
\hline & $\mathrm{O}_{2} \mathrm{Hb}$ & HHb & tHb & HR & LAC & RPE \\
\hline & $\mathrm{R}=0.03$ & $\mathrm{R}=0.09$ & $\mathrm{R}=0.03$ & $\mathrm{R}=0.33$ & $\mathrm{R}=0.22$ & $\mathrm{R}=0.16$ \\
\hline EEG & $P=0.75$ & $P=0.28$ & $P=0.67$ & $P<0.001$ & $P=0.01$ & $P=0.11$ \\
\hline HR & $\mathrm{R}=0.43$ & $\mathrm{R}=0.52$ & $\mathrm{R}=0.58$ & $\mathrm{n} / \mathrm{a}$ & $\mathrm{R}=0.63$ & $\mathrm{R}=0.76$ \\
\hline HR & $P<0.001$ & $P<0.001$ & $P<0.001$ & $\mathrm{n} / \mathrm{a}$ & $P<0.001$ & $P<0.001$ \\
\hline LAC & $\begin{array}{l}\mathrm{R}=0.31 \\
P<0.001\end{array}$ & $\begin{array}{l}\mathrm{R}=0.28 \\
P=0.001\end{array}$ & $\begin{array}{l}\mathrm{R}=0.35 \\
P<0.001\end{array}$ & $\begin{array}{l}\mathrm{R}=0.63 \\
P<0.001\end{array}$ & $\mathrm{n} / \mathrm{a}$ & $\begin{array}{l}\mathrm{R}=0.65 \\
P<0.001\end{array}$ \\
\hline RPE & $\begin{array}{l}\mathrm{R}=0.40 \\
P<0.001\end{array}$ & $\begin{array}{l}\mathrm{R}=0.31 \\
P=0.002\end{array}$ & $\begin{array}{l}\mathrm{R}=0.48 \\
P<0.001\end{array}$ & $\begin{array}{l}\mathrm{R}=0.76 \\
P<0.001\end{array}$ & $\begin{array}{l}\mathrm{R}=0.65 \\
P<0.001\end{array}$ & $\mathrm{n} / \mathrm{a}$ \\
\hline HHb & $\begin{array}{l}\mathrm{R}=0.04 \\
P=0.66\end{array}$ & $\mathrm{n} / \mathrm{a}$ & $\begin{array}{l}\mathrm{R}=0.33 \\
P<0.001\end{array}$ & $\begin{array}{l}\mathrm{R}=0.52 \\
P<0.001\end{array}$ & $\begin{array}{l}\mathrm{R}=0.28 \\
P=0.001\end{array}$ & $\begin{array}{l}\mathrm{R}=0.31 \\
P=0.002\end{array}$ \\
\hline tHb & $\begin{array}{l}\mathrm{R}=0.87 \\
P<0.001\end{array}$ & $\begin{array}{l}\mathrm{R}=0.33 \\
P<0.001\end{array}$ & $\mathrm{n} / \mathrm{a}$ & $\begin{array}{l}\mathrm{R}=0.58 \\
P<0.001\end{array}$ & $\begin{array}{l}\mathrm{R}=0.35 \\
P<0.001\end{array}$ & $\begin{array}{l}\mathrm{R}=0.48 \\
P<0.001\end{array}$ \\
\hline
\end{tabular}

\section{Discussion}

This study aimed to show hemodynamic and electro-cortical changes in parallel within the left and right hemispheres of the PFC during an incremental bicycle exercise test under normoxic and hypoxic conditions for correlation. For haemodynamic parameters, significant changes with exercise intensity as well as differences between conditions and hemispheres were found. Electro-cortical activity within the PFC did not result in differences between conditions and hemispheres. No correlations between hemodynamic and electro-cortical parameters were found.

\subsection{Heart Rate, Lactate Concentration and Ratings of Perceived Exertion}

Regarding absolute intensity, exercise test ended earlier under hypoxic than normoxic conditions (decrease of about 19.1\%) as expected [4, 27]. After adjusting the data to the last three exercise stages respectively for both conditions instead of regarding absolute intensity levels, heart rate, lactate concentration and ratings of perceived exertion did not differ between conditions. Thus, the same intensity under hypoxic conditions is on physical and mental basis more demanding than normoxic conditions. Heart rate and ratings of perceived exertion values indicate that exercise has been discontinued shortly before reaching the subjective maximum, which is due to the maximum heart rate limit of 180 beats/minute. This should be kept in mind for the following discussion.

\subsection{NIRS}

All together, NIRS parameters are in line with previous findings demonstrating a clearly better central oxygenation situation under normoxic, compared to hypoxic conditions within the PFC during bicycling exercise at moderate to submaximal exercise intensity [2, 3, 8-11]. Therefore, replicated NIRS results will not be discussed in detail and the following discussion will concentrate on relevant and new findings.

tHb: The clear lower haemoglobin concentration - thus blood volume-under hypoxic condition within both hemispheres starting with onset of exercise and remaining up to post exercise is remarkable (please note that measurements have been baseline corrected - that is why pre measurements are on same level). This demonstrates that less blood is delivered to the PFC probably in favour of other cerebral and peripheral regions, which are of higher importance at that time to maintain exercise and vital tasks such as the working muscles, motor cortex, basal ganglia or thalamus, and brain stem [2, 6, 28-30].

Moreover, during exercise under normoxic condition, the right hemisphere seems to be fed at higher extent compared to the left hemisphere, although marginally non-significant $(P=0.068)$. Prior 
studies also report of higher right-sided $\mathrm{O}_{2} \mathrm{Hb}$ and $\mathrm{tHb}$ concentration for normoxic and hypoxic conditions [3]. Higher $\mathrm{O}_{2} \mathrm{Hb}$ and tHb concentration can either be an indicator of higher neuronal activity or higher blood supply. Here, higher activity within the left hemisphere was found in normoxia, which supports the notion that higher brain activity is not the reason for increasing $\mathrm{tHb}$ (and $\mathrm{O}_{2} \mathrm{Hb}$ ). Consequently, the higher $\mathrm{O}_{2} \mathrm{Hb}$ values in normoxia seem to be a result of higher blood supply $(\mathrm{tHb})$ to the right hemisphere and not be caused by higher right-sided PFC activation, as speculated before by Subudhi [3].

$\mathrm{O}_{2} \mathrm{Hb}$ : Regarding normoxia, the higher the workload the more oxygen is present within the PFC, which seems to be mainly based on higher blood volume/flow as indicated by parallel increase of tHb and HR values [31]. Under hypoxic conditions, a far lower $\mathrm{O}_{2} \mathrm{Hb}$ concentration was found during exercise and after exercise compared to normoxia. Physical load under hypoxic conditions results in a high oxygen deficit, which is revealed by the initial dip of $\mathrm{O}_{2} \mathrm{HB}$ values during exercise compared to "pre" although tHb sustained or increased. The continuative increase post-exercise indicates that oxygen deficit is partly cleared by the decreasing oxygen consumption without exercise, as reflected by the decrease in $\mathrm{HHb}$ concentration and a constant $\mathrm{tHb}$ level. The expected decrease shortly before the end of the exercise test, as shown in prior NIRS studies [6,9], could not be found here, which is due to the fact that more than half of the tests were not carried out until subjective exhaustion, but stopped at heart rate of 180 beats/minute.

$\mathrm{HHb}$ : Striking is the difference between conditions with far higher $\mathrm{HHb}$ during exercise under hypoxic conditions. The higher $\mathrm{HHb}$ concentration under hypoxic conditions reflects the impaired oxygen situation as seen in $\mathrm{O}_{2} \mathrm{Hb}$ within the $\mathrm{PFC}$, due to lesser blood delivery $(\mathrm{tHb})$ and lower oxygen fraction of the air. Post exercise, $\mathrm{HHb}$ in hypoxia dropped dramatically and reached the level of post measurements in normoxia, which means that less oxygen is consumed when no physical load is present and the brain is able to clear the oxygen deficit present during exercise.

\section{$4.3 E E G$}

Regarding electro-cortical activity of the PFC, higher cortical current density during exercise compared to pre and post exercise was found. Earlier studies did not find elevations of PFC activity with bicycling intensity [19], but these included the whole prefrontal cortex and not only BA10 and BA46. Both brain regions are known to be involved into cognitive processes like attention, learning and memory in regard of motivation, decision-making, conflict monitoring, integration of separate cognitive operation outcomes in pursuit of higher behavioural goals and inhibition of non-appropriate actions [32-39]. Accordingly, these areas might be increasingly activated with intensity and progression of the test and fatigue, when it more and more comes to thoughts and motivational processes in regard to how far the person wants to and is able to go. Further studies are required to explore the role of the PFC hemispheres in regard to fatigue, especially at the moment of withdrawal/exhaustion.

Different to what was identified in the NIRS data, no main effect for condition or hemisphere was found in EEG. Brain cortical activity within the PFC was slightly lower in hypoxia than normoxia almost throughout the test and the elevation of PFC activity in hypoxia is not as distinct as in normoxia, especially within the left hemisphere. However, the missing CONDITION effect for EEG data does not allow concluding onto a general impaired PFC activity in hypoxia, as it would be expected based on the NIRS results. Accordingly, lower PFC oxygenation (lower $\mathrm{O}_{2} \mathrm{Hb}$, higher $\mathrm{HHb}$ ) does not necessarily mean lower PFC activity as assumed in previous investigations [3, $6,9,12-14]$. Additionally, the missing main effect for HEMISPHERE regarding EEG but existing hemispheric difference in NIRS suggest that EEG and NIRS results cannot be directly transferred and that 
hemodynamic changes assessed by NIRS might be influenced by other/additional factors than brain cortical activity.

\subsection{Correlation}

The fact that the multiple regression analysis did not show any correlation between EEG and NIRS parameters support the assumption that NIRS parameters of the PFC during intensive exercise are not primarily dependent on brain-cortical activity. The oxygenation situation does not truly reflect electro-cortical activity in exercise studies. Exercise per se is known to go along with fluid shifts and changes in blood volume distributions, as well as blood pressure and blood flow [40-42]. The systemic effect of exercise with higher perfusion is assumed to overlay the relatively small effects of active neurons on hemodynamic situation. It is even more evident when taking the strong positive correlation of $\mathrm{O}_{2} \mathrm{Hb}$ with heart rate into account. The data highlights that interpretation of NIRS data- in regard to neural activation and exercise- should be done with caution (it is not as easy as pulling a white rabbit out of a hat). Although the data demonstrate clear effects, results should be validated because of the limited number of participants.

\section{Conclusion}

Oxygenation of the brain is impaired during exercise in hypoxia, whereas brain cortical activity is not. No correlation between hemodynamic and electro-cortical parameters were found, which is supposed to be due to the high systemic hemodynamic changes predominating the NIRS signal and overlaying changes attributable to neural activity. The results shed light on the correlation between two different methods for brain imaging during high-intensity exercise and emphasize that transferability of results between methods have to be done with caution. It is recommended to use additional assessments like blood flow measurements, blood pressure, heart rate etc. are required to correct the
NIRS data or to use supplementary brain assessment techniques, such as EEG or MRI, for correlation.

\section{Acknowledgements}

The authors wish to thank the participants for their time and effort, and Anna Berger and FabianSchürg for their help with data collection. The study was founded by an internal research grant (\#920044) of the German Sport University Cologne. The authors declare that they have no conflict of interest.

\section{References}

[1] Villringer, A., and Obrig, H. 2002. "Near-infrared Spectroscopy and Imaging." In Brain Mapping: The Methods (2nd ed.), edited by Toga, A. W., and Mazziotta, J. C., Amsterdam: Academic, 141-58.

[2] Subudhi, A. W., Dimmen, A. C., and Roach, R. C. 2007. "Effects of Acute Hypoxia on Cerebral and Muscle Oxygenation during Incremental Exercise." J. Appl. Physiol. 103 (1): 177-83.

[3] Subudhi, A. W., Miramon, B. R., Granger, M. E., and Roach, R. C. 2009. "Frontal and Motor Cortex Oxygenation during Maximal Exercise in Normoxia and Hypoxia.” J. Appl. Physiol. 106 (4): 1153-8.

[4] Patrick Neary, J., Roberts, A. D., Leavins, N., Harrison, M. F., Croll, J. C., and Sexsmith, J. R. 2008. "Prefrontal Cortex Oxygenation during Incremental Exercise in Chronic Fatigue Syndrome." Clin. Physiol. Funct. Imaging 28 (6): 364-72.

[5] Billaut, F., Davis, J. M., Smith, K. J., Marino, F. E., and Noakes, T. D. 2010. "Cerebral Oxygenation Decreases but Does Not Impair Performance During Self-paced, Strenuous Exercise.” Acta physiologica 198 (4): 477-86.

[6] Rupp, T., and Perrey, S. 2008. "Prefrontal Cortex Oxygenation and Neuromuscular Responses to Exhaustive Exercise." Eur. J. Appl. Physiol. 102 (2): 153-63.

[7] Peltonen, J. E., Kowalchuk, J. M., Paterson, D. H., DeLorey, D. S., duManoir, G. R., Petrella, R. J., and Shoemaker, J. K. 2007. "Cerebral and Muscle Tissue Oxygenation in Acute Hypoxic Ventilatory Response Test." Respir. Physiol. Neurobiol. 155 (1): 71-81.

[8] Millet, G. Y., Muthalib, M., Jubeau, M., Laursen, P. B., and Nosaka, K. 2012. "Severe Hypoxia Affects Exercise Performance Independently of Afferent Feedback and Peripheral Fatigue.” J. Appl. Physiol. 112 (8): 1335-44.

[9] Rupp, T., and Perrey, S. 2009. "Effect of Severe Hypoxia on Prefrontal Cortex and Muscle Oxygenation Responses at Rest and during Exhaustive Exercise." Adv. Exp. Med. Biol. 645: 329-34. 
[10] Subudhi, A. W., Lorenz, M. C., Fulco, C. S., and Roach, R. C. 2008. "Cerebrovascular Responses to Incremental Exercise during Hypobaric Hypoxia: Effect of Oxygenation on Maximal Performance." Am. J. Physiol. Heart Circ. Physiol. 294 (1): H164-71.

[11] Amann, M., Romer, L. M., Subudhi, A. W., Pegelow, D. F., and Dempsey, J. A. 2007. "Severity of Arterial Hypoxaemia Affects the Relative Contributions of Peripheral Muscle Fatigue to Exercise Performance in Healthy Humans." J. Physiol. 581 (Pt 1): 389-403.

[12] Ekkekakis, P. 2009. "Illuminating the Black Box: Investigating Prefrontal Cortical Hemodynamics during Exercise with Near-infrared Spectroscopy." J. Sport Exerc. Psychol. 31 (4): 505-53.

[13] Shibuya, K., \& Tachi, M. 2006. "Oxygenation in the Motor Cortex during Exhaustive Pinching Exercise." Respir. Physiol. Neurobiol. 153 (3): 261-6.

[14] St Clair Gibson, A., and Noakes, T. D. 2004. "Evidence for Complex System Integration and Dynamic Neural Regulation of Skeletal Muscle Recruitment during Exercise in Humans.” Br. J. Sports Med. 38 (6): 797-806.

[15] Rasmussen, P., Nielsen, J., Overgaard, M., Krogh-Madsen, R., Gjedde, A., Secher, N. H., and Petersen, N. C. 2010. "Reduced Muscle Activation during Exercise Related to Brain Oxygenation and Metabolism in Humans." The Journal of Physiology 588 (Pt 11): 1985-95.

[16] Amann, M., and Calbet, J. A. 2008. "Convective Oxygen Transport and Fatigue.” J. Appl. Physiol. 104 (3): 861-70.

[17] Seifert, T., Rasmussen, P., Secher, N. H., and Nielsen, H. B. 2009. "Cerebral Oxygenation Decreases during Exercise in Humans with Beta-adrenergic Blockade." Acta physiologica 196 (3): 295-302.

[18] Crabbe, J. B., and Dishman, R. K. 2004. "Brain Electrocortical Activity during and after Exercise: a Quantitative Synthesis.” Psychophysiology 41 (4): 563-74.

[19] Brummer, V., Schneider, S., Struder, H. K., and Askew, C. D. 2011. "Primary Motor Cortex Activity is Elevated with Incremental Exercise Intensity." Neuroscience 181: 150-62.

[20] Obrig, H., and Villringer, A. 2003. "Beyond the Visible-imaging the Human Brain with Light." J. Cereb Blood Flow Metab. 23 (1): 1-18.

[21] Schneider, S., and Strüder, H. K. 2009. "Monitoring Effects of Acute Hypoxia on Brain Cortical Activity by Using Electromagnetic Tomography." Behav Brain Res. 197 (2): 476-80.

[22] Borg, G. A. 1982. "Psychophysical Bases of Perceived Exertion." Med. Sci. Sports Exerc. 14 (5): 377-81.

[23] Abeln, V., Harig, A., Knicker, A., Vogt, T., and Schneider, S. 2013. "Brain-imaging during an Isometric Leg Extension Task at Graded Intensities." Front Physiol. 4: 296.
[24] Duncan, A., Meek, J. H., Clemence, M., Elwell, C. E., Fallon, P., Tyszczuk, L., Cope, M., Delpy, D. T. 1996. "Measurement of Cranial Optical Path Length as a Function of Age using Phase Resolved Near Infrared Spectroscopy." Pediatr. Res. 39 (5): 889-94.

[25] Hollmann, W., and Venrath, H. 1966. "The Behavior of the Cardiopulmonary System and the Skeletal Muscular Strength in Loads with Various Degrees of Oxygen Content of the Air." Schweiz Z Sportmed 14 (1): 27-34.

[26] Buck, A., Schirlo, C., Jasinksy, V., Weber, B., Burger, C., von Schulthess, G. K., Koller, E. A., and Pavlicek, V. 1998. "Changes of Cerebral Blood Flow during Short-term Exposure to Normobaric Hypoxia." J. Cereb. Blood Flow Metab. 18 (8): 906-10.

[27] Binks, A. P., Cunningham, V. J., Adams, L., and Banzett, R. B. 2008. "Gray Matter Blood Flow Change is Unevenly Distributed during Moderate Isocapnic Hypoxia in Humans." J. Appl. Physiol. 104 (1): 212-7.

[28] Ainslie, P. N., Barach, A., Murrell, C., Hamlin, M., Hellemans, J., and Ogoh, S. 2007. "Alterations in Cerebral Autoregulation and Cerebral Blood Flow Velocity during Acute Hypoxia: Rest and Exercise." American Journal of Physiology. Heart and Circulatory Physiology 292 (2): H976-83.

[29] Gonzalez-Alonso, J., Dalsgaard, M. K., Osada, T., Volianitis, S., Dawson, E. A., Yoshiga, C. C., and Secher, N. H. 2004. "Brain and Central Haemodynamics and Oxygenation during Maximal Exercise in Humans." $J$. Physiol. 557 (Pt 1): 331-42.

[30] Allman, J., Hakeem, A., and Watson, K. 2002. "Two Phylogenetic Specializations in the Human Brain." Neuroscientist 8 (4): 335-46.

[31] Kuhl, B. A., Dudukovic, N. M., Kahn, I., and Wagner, A. D. 2007. "Decreased Demands on Cognitive Control reveal the Neural Processing Benefits of Forgetting." Nat. Neurosci. 10 (7): 908-14.

[32] Moghaddam, B., and Homayoun, H. 2008. "Divergent Plasticity of Prefrontal Cortex Networks." Neuropsychopharmacology 33 (1): 42-55.

[33] Ramnani, N., and Owen, A. M. 2004. "Anterior Prefrontal Cortex: Insights into Function from Anatomy and Neuroimaging." Nat. Rev. Neurosci. 5 (3): 184-94.

[34] Schiffer, A. M., Ahlheim, C., Ulrichs, K., and Schubotz, R. I. 2012. "Neural Changes When Actions Change: Adaptation of Strong and Weak Expectations." Hum. Brain Mapp. 34 (7): 1713-27.

[35] Dalley, J. W., Cardinal, R. N., and Robbins, T. W. 2004. "Prefrontal Executive and Cognitive Functions in Rodents: Neural and Neurochemical Substrates." Neurosci. Biobehav. Rev. 28 (7): 771-84.

[36] Babiloni, C., Vecchio, F., Bares, M., Brazdil, M., Nestrasil, I., Eusebi, F., Rossini, P.M., and Rektor, I. 2008. 


\section{Exercise in Normoxia and Hypoxia-A Combined EEG/NIRS Study}

"Functional Coupling Between Anterior Prefrontal Cortex (BA10) and Hand Muscle Contraction during Intentional and Imitative Motor Acts." Neuroimage 39 (3): 1314-23.

[37] Wriessnegger, S. C., Bauernfeind, G., Schweitzer, K., Kober, S., Neuper, C., and Muller-Putz, G. R. 2012. "The Interplay of Prefrontal and Sensorimotor Cortices during Inhibitory Control of Learned Motor Behavior." Front Neuroeng 5: 17.

[38] Secher, N. H., and Amann, M. 2012. "Human Investigations into the Exercise Pressor Reflex."
Experimental Physiology 97 (1): 59-69.

[39] Ogoh, S., and Ainslie, P. N. 2009. "Cerebral Blood Flow during Exercise: Mechanisms of Regulation." Journal of Applied Physiology 107 (5): 1370-80.

[40] Luft, A. R., Smith, G. V., Forrester, L., Whitall, J., Macko, R. F., Hauser, T. K., Goldberg, A. P., and Hanley, D. F. 2002. "Comparing Brain Activation Associated with Isolated Upper and Lower Limb Movement across Corresponding Joints." Human Brain Mapping 17 (2): 131-40. 